\title{
Implementation of interval walking training in patients with type 2 diabetes in Denmark: rationale, design, and baseline characteristics
}

This article was published in the following Dove Press journal:

Clinical Epidemiology

8 June 2016

Number of times this article has been viewed

\author{
Mathias Ried-Larsen ${ }^{1-3}$ \\ Reimar W Thomsen ${ }^{2,4}$ \\ Klara Berencsi ${ }^{4}$ \\ Cecilie F Brinkløv 1,5 \\ Charlotte Brøns ${ }^{1,5}$ \\ Laura S Valentiner ${ }^{1,6}$ \\ Kristian Karstoft ${ }^{1,3}$ \\ Henning Langberg ${ }^{1,6}$ \\ Allan A Vaag ${ }^{1,2,5}$ \\ Bente K Pedersen ${ }^{1,3}$ \\ Jens S Nielsen ${ }^{7}$
}

'Department of Infectious Diseases, Centre for Physical Activity

Research, Rigshospitalet, University

of Copenhagen, Copenhagen,

${ }^{2}$ The Danish Diabetes Academy,

Odense University Hospital,

Odense, ${ }^{3}$ Department of Infectious

Diseases, Centre of Inflammation and Metabolism, Rigshospitalet, University of Copenhagen,

Copenhagen, ${ }^{4}$ Department of Clinical Epidemiology, Aarhus University Hospital, Aarhus Nord, ${ }^{5}$ Department of Endocrinology (Diabetes and Metabolism), Rigshospitalet, University of Copenhagen, ${ }^{6}$ CopenRehab, Department of Public Health, Section of Social Medicine, University of Copenhagen, Copenhagen, ${ }^{7}$ Department of Endocrinology, Odense University Hospital, Odense, Denmark

Correspondence: Mathias Ried-Larsen Department of Infectious Diseases, Centre for Physical Activity Research, Rigshospitalet, University of Copenhagen, Blegdamsvej 9, 2100 Copenhagen,

Denmark

Tel +4535450699

Email Mathias.ried-larsen@regionh.dk
Abstract: Promoting physical activity is a first-line choice of treatment for patients with type 2 diabetes (T2D). However, there is a need for more effective tools and technologies to facilitate structured lifestyle interventions and to ensure a better compliance, sustainability, and health benefits of exercise training in patients with T2D. The InterWalk initiative and its innovative application (app) for smartphones described in this study were developed by the Danish Centre for Strategic Research in T2D aiming at implementing, testing, and validating interval walking in patients with T2D in Denmark. The interval walking training approach consists of repetitive 3-minute cycles of slow and fast walking with simultaneous intensity guiding, based on the exercise capacity of the user. The individual intensity during slow and fast walking is determined by a short initial self-conducted and audio-guided fitness test, which combined with automated audio instructions strives to motivate the individual to adjust the intensity to the predetermined individualized walking intensities. The InterWalk app data are collected prospectively from all users and will be linked to the unique Danish nationwide databases and administrative registries, allowing extensive epidemiological studies of exercise in patients with T2D, such as the level of adherence to InterWalk training and long-term effectiveness surveys of important health outcomes, including cardiovascular morbidity and mortality. Currently, the InterWalk app has been downloaded by $>30,000$ persons, and the achieved epidemiological data quality is encouraging. Of the 9,466 persons providing personal information, $80 \%$ of the men and $62 \%$ women were overweight or obese (body mass index $\geq 25$ ). The InterWalk project represents a contemporary technology-driven public health approach to monitor reallife exercise adherence and to propagate improved health through exercise intervention in T2D and in the general population.

Keywords: exercise, telemedicine, cell phones

\section{Background}

It is well documented that increasing physical activity levels is an effective first-line treatment for patients with type 2 diabetes (T2D). ${ }^{1,2}$ There is convincing evidence to support important clinical benefits of supervised aerobic training in patients with T2D on, for example, hemoglobin A1c level, lipid profile, quality of life, physical fitness, and other parameters. ${ }^{3}$ However, a recent meta-analysis concluded that while performance of supervised exercise was effective in improving glycemic control in T2D patients, advice about physical activity alone was not effective on this particular outcome. ${ }^{4}$ As the number of people with T2D worldwide is estimated to reach 439-552 million by $2030,5,6$ fully supervised long-term training programs for all T2D patients will be very costly and thus unrealistic. Instead, there is a need for improved physical activity 
rehabilitation programs with high adherence that may be implemented in large cohorts of patients with T2D with little or no supervision.

More than $60 \%$ of the Danish households and $55 \%$ of all American adults currently have a smartphone. ${ }^{7,8}$ Using a smartphone as a vehicle to promote physical activity thus renders a unique, feasible, and cost-effective opportunity to improve rehabilitation in a larger proportion of the population with T2D. Accordingly, we developed a smartphone application (app) - InterWalk - to promote a sustainable increase in the physical activity level in the Danish population with T2D.

\section{Aims}

This article describes the rationale behind and the development and design of the InterWalk smartphone app. Furthermore, this article outlines the planned scientific evaluation of the nationwide InterWalk implementation.

\section{Methods and design}

\section{Reflections and perspectives related to} interval walking training in patients with T2D

An increasing body of evidence suggests that the intensity and modality (eg, interval vs continuous training) of physical activity play an important role for improving clinically relevant outcomes in $\mathrm{T} 2 \mathrm{D}$, independent of the total physical activity volume. ${ }^{9}$ One of these new modalities is interval walking training (IWT), developed and evaluated in several studies of healthy, elderly Japanese people. ${ }^{10-13}$ IWT consists of repetitive cycles of 3 minutes of fast and slow walking during free living guided by an accelerometer-based portable training device (JD Mate; Kissei Comtec Co., Ltd, Matsumoto, Japan). ${ }^{14}$ These initial studies showed beneficial effects on body composition, blood pressure, physical fitness, and strength measurements, combined with a high level of adherence $(>95 \%)$ over a period of 4 months in a healthy elderly population $(\mathrm{N}>700)$, despite a minimum of supervision. ${ }^{12,13}$

With its individualized physical activity intensity, IWT was potentially a feasible intervention for T2D patients. Thus, Karstoft et $\mathrm{al}^{15}$ examined the feasibility of implementing the IWT modality among patients with T2D in a free-living environment and compared it to energy expenditure-matched continuous walking training and to a control group. In this 4-month randomized controlled trial, IWT, but not continuous walking training, improved key metabolic parameters relevant for patients with T2D. ${ }^{15}$ Indeed, IWT resulted in a $16 \%$ increase in physical fitness level, a $3 \mathrm{~kg}$ decrease in body fat mass, and a decrease in hyperglycemic levels without accompanying hypoglycemic episodes. Moreover, this was achieved with a compliance rate of $\sim 85 \%$ and very low loss to follow-up.

At the end of the clinical trial, we administered a brief questionnaire to acquire information about the participants' motivation to continue the training intervention (Figure 1) and what possible factors could motivate them to continue (Figure 2). At the 1-year follow-up, we asked both the continuous walking group and the interval walking group about their motivation to continue the training intervention (Figure 3). The encouraging feedback along with the clinically significant results inspired us to move forward with regard to implementing the interval walking modality in rehabilitation programs for patients with T2D.

The development process of the InterWalk app (Visuallogic, Nyborg, Denmark) is described in Figure 4. To develop a setting and to test the vehicle to deliver IWT, we implemented the JD Mate in a municipality-based rehabilitation program. During this iteration, we identified several barriers to succeed with large-scale implementation. The barriers included difficult data transfer, thus complicating the deliverance of feedback to the user (upload is performed using a computer), usability (the device language was Japanese), and reliability ( $\sim 15 \%$ of all devices malfunctioned during the iteration). However, the pattern of motivational factors was similar to what we had observed in the clinical trial (Figure 2).

Based on this, we developed a smartphone app (InterWalk) with features similar to those of the JD Mate using a participatory design. Briefly, the participatory design proposes inclusion of all key stakeholders including the users during the design of the intervention. ${ }^{16,17}$ First, we identified

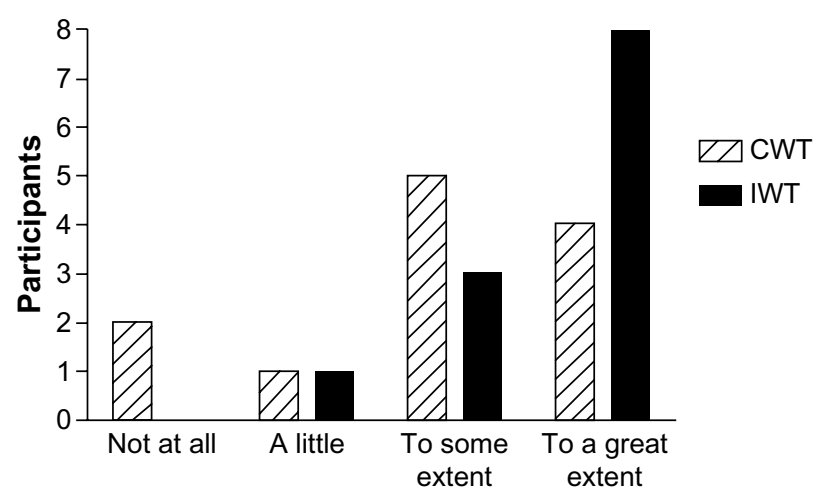

Figure I Degree of motivation to continue the training intervention among participants at the end of a 4-month clinical trial assessing the efficacy of interval walking training.

Notes: Participants were randomized to continuous walking training (CWT) or interval walking training (IWT). The participants were asked to rate the degree of motivation. 


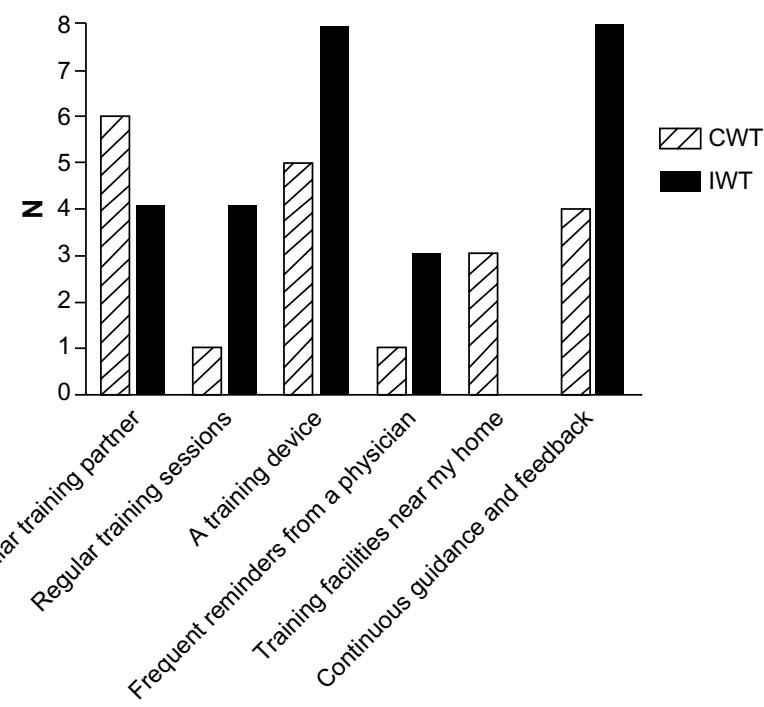

Figure 2 Level of agreement with statements regarding what would motivate the participants to continue to perform walking training at the end of a 4-month clinical trial assessing the efficacy of interval walking training.

Notes: Participants were randomized to continuous walking training (CWT) or interval walking training (IWT).

key stakeholders in the rehabilitation of persons with T2D in Denmark ${ }^{18}$ and arranged a series of workshops. The key stakeholders were identified as persons with T2D, rehabilitation therapists from the Danish municipalities, personnel at hospital-based outpatient clinics, and the Danish Diabetes Association. Initially, the JD Mate user feedback was used to develop two different app design mockups. The designs were tested in a municipality rehabilitation setting, in a peerto-peer setting in collaboration with the Danish Diabetes Association, and in a hospital outpatient clinic ( $\mathrm{n}=17)$. Feedback from the focus group interviews was incorporated in the design of the app (Cosmographic A/S, Denmark). Prior to release in AppStore, pilot testing of the beta version was

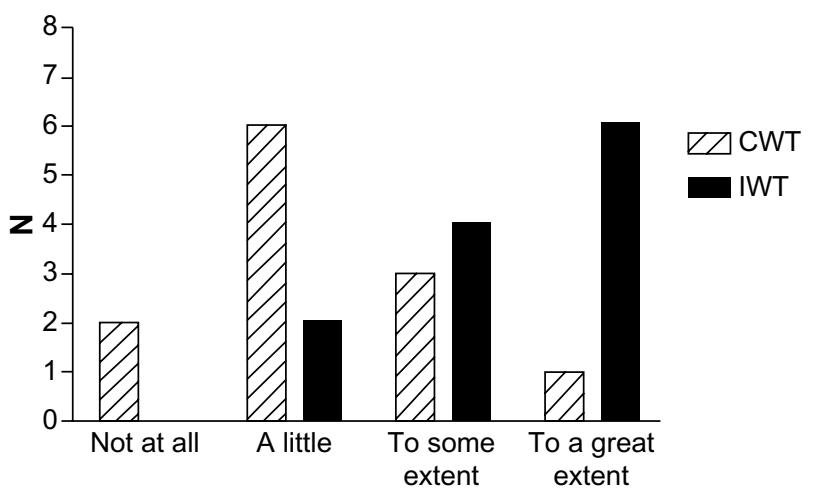

Figure 3 Degree of motivation to continue the training intervention among participants at I-year follow-up following the 4-month clinical trial assessing the efficacy of interval walking training.

Notes: Participants were randomized to continuous walking training (CWT) or interval walking training (IWT). The participants were asked to rate the degree of motivation. performed in the municipality $\mathrm{T} 2 \mathrm{D}$ rehabilitation program $(\mathrm{n}=18)$, and feedback was used to further adjust app functionalities, including increased user feedback and graphical presentation of the exercise history (Figure 4).

The app Version 1.0 was released in AppStore in November 2013, and a subsequent test of unsupported use was performed and evaluated by observations and focus group interviews $(n=25)$ in collaboration with the Danish Diabetes Association. The participants found the InterWalk app to be manageable and meaningful in their everyday life. However, there was a need for an extension of the help function, an increased focus on retaining the quality of the exercise, and an automated data upload function from iPods. Finally, based on the user feedback from the therapists in three Danish municipalities, the current version was updated with an increased volume of the audio and voice feedback. This version of InterWalk was released to AppStore in December 2015. This trial has been registered with the NCT under number NCT01234155.

\section{Nationwide data linking of the InterWalk training data}

The Danish civil registration number (Central Personal Registry [CPR] number) is a unique personal identifier, provided to all Danish residents at birth or migration into Denmark. The CPR number is used to register each citizen's primary and secondary health care contacts, redeemed prescription medications, education and income statistics, and other information from the Danish health and administrative databases. ${ }^{19}$ Providing the CPR number is mandatory in order to use the InterWalk app. The CPR number is automatically hashed (see Data logging and storage) and uploaded to a central server, together with collected training data after each test and training session. Using the CPR number in InterWalk allows for subsequent secure data linkage in later research projects, with access to baseline and follow-up health data for each individual. ${ }^{20}$ The CPR number is thus pivotal in order to study the implementation of the InterWalk app and the long-term health effects of InterWalk usage.

\section{InterWalk design and content description}

The InterWalk app is developed for use on smartphones with the Apple iPhone Operating System or Android Operating System (Version 2.3 or higher for LG and HTC) operating systems and consists of the following subcomponents: an IWT program using audio guidance to help the user through the session, a fitness test used for setting the intensity during IWT, an IWT history for the user to keep track of progress, logging of accelerometer, and Global Positioning System 


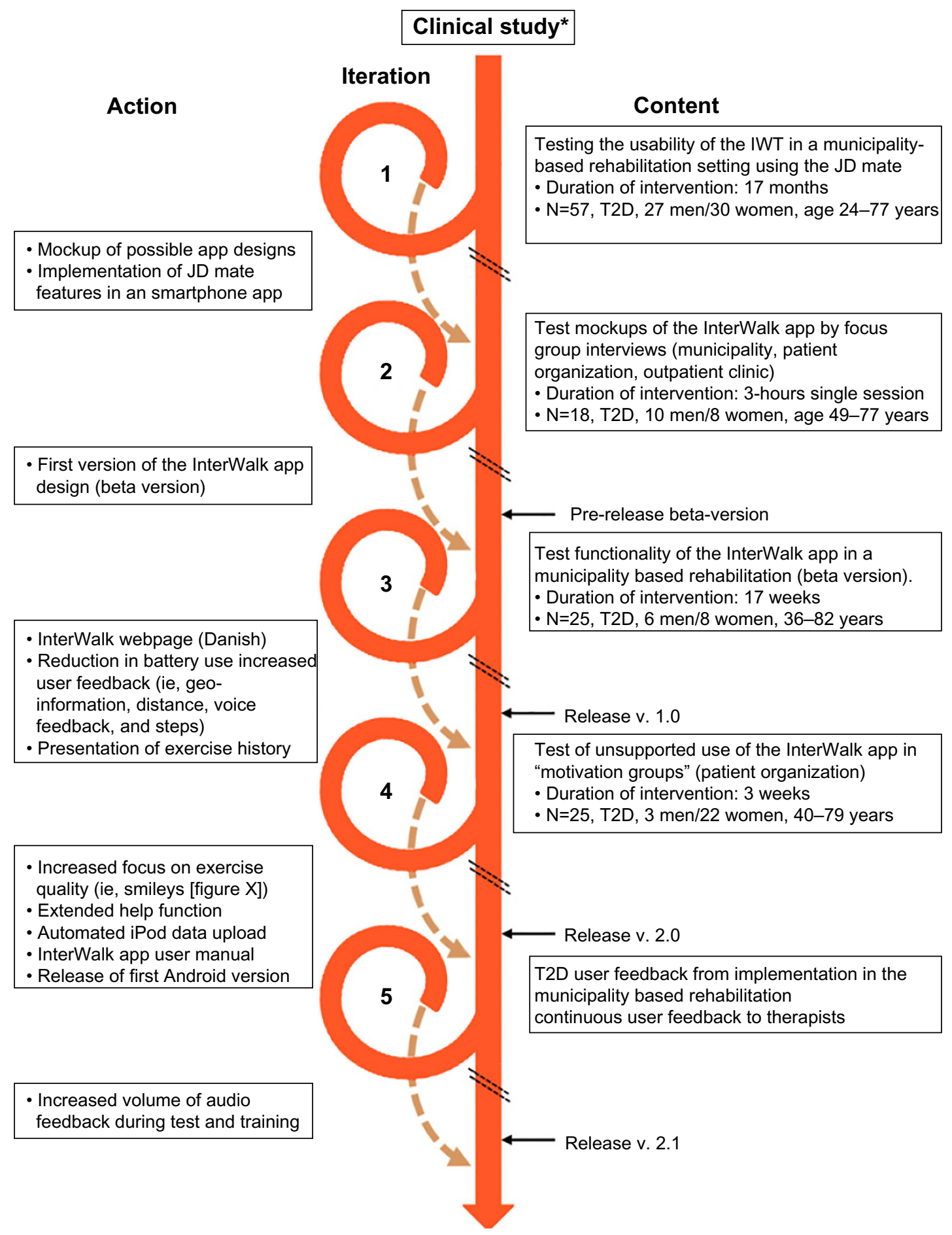

Figure 4 Development process of the InterWalk app after a 4-month clinical trial $\left(^{*}\right)$ assessing the efficacy of interval walking training. Abbreviations: IWT, interval walking training; T2D, type 2 diabetes.

(GPS) data obtained during the IWT sessions, and an individualized fitness test. The subcomponents are described in detail later. Upon the first use of the app, a user profile needs to be completed (CPR number, sex, body weight in kilograms, and height in centimeters). Before engaging in IWT, the app recommends completion of a fitness test. This will set the individual user exercise intensity during IWT.
The layout of the front page of the InterWalk app is depicted in Figure 5A.

\section{Interval walking training}

IWT consists of repeated cycles of 3 minutes of slow and fast walking. The individualized walking intensity for slow and fast walking during IWT is calculated from accelerometer 
A

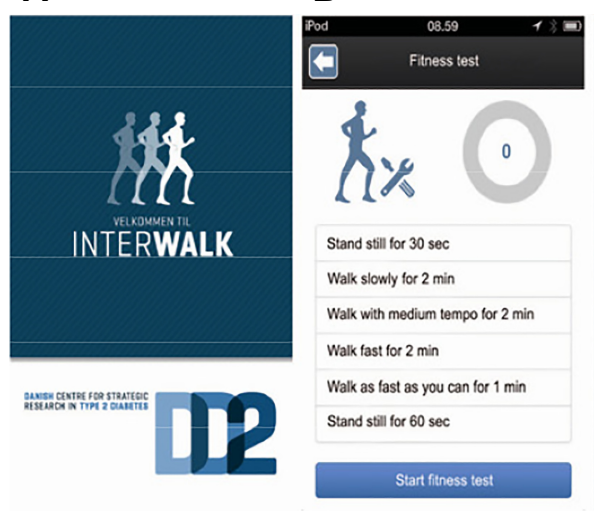

C

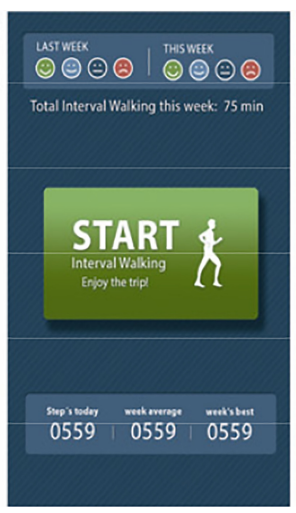

D

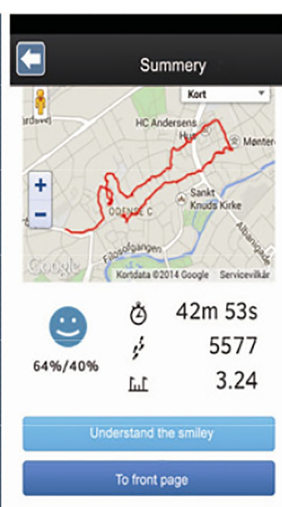

E

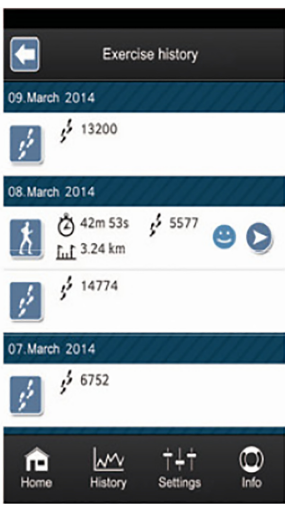

Figure 5 Layout of the InterWalk app (translated from Danish).

Notes: (A) The front page and the InterWalk app logo, (B) the protocol for the fitness test, (C) the start page including the user progression (smileys) across the current and previous weeks, (D) the immediate feedback after an InterWalk session, and (E) the historical InterWalk user data.

data collected during the fitness test. It is possible to do IWT without a prior fitness test; however, in that case, walking intensity audio guidance will not be available. As a large proportion of the target group is assumed to be untrained, we have proposed an in-app progression in the "help" function specifying the training duration per session and training frequency for beginners (10-20 minutes, three times per week), intermediate users (20-30 minutes, three times per week), and advanced users ( $>30$ minutes, more than three times per week). The duration and frequency should be increased in steps of 10 minutes until the users are able to complete five sessions per week of 60 minutes per session. This progression in training volume was determined based on user interviews and expert consensus.

\section{The fitness test}

The test is a standardized 7-minute walking test protocol. The user is encouraged on the start-up screen of the app to perform the test before engaging in IWT for the first time. The protocol consists of four consecutive walking intervals: slow ( 2 minutes), intermediate ( 2 minutes), fast ( 2 minutes), and very fast walking ( 1 minute). Prior to the first and immediately after the last walking intensity, users are instructed to stand still for 30 seconds and 60 seconds, respectively, in order for the app to detect a valid GPS signal (protocol shown in Figure 5B). The walking intensity is self-selected, that is, what "slow walking," "fast walking," and so on imply is defined by the user. The onset of the test and each alternation in walking intensity are audio guided, thus automatically instructing the user to start and subsequently change the walking intensity according to the above-described walking intensities. The training start page is depicted in Figure 5C. Upon use, it is recommended on the app screen that the smartphone is placed near the waistline or in the side pockets of the pants while performing the test and IWT.

\section{Walking intensity during IWT}

The IWT intensity is based on the accelerometer recording during the fitness test. The smartphone on-board accelerometer is recording acceleration with a $100 \mathrm{~Hz}$ sampling frequency. Acceleration from $x, y$, and $z$ axes is used to calculate the vector magnitude as the square root of the summed squared acceleration from the $x, y$, and $z$ axes. Subsequently, the magnitude is averaged across 10 seconds during IWT (10-second epochs) and 30 seconds during the individual fitness test (30-second epochs). The slow walking cutoff intensity during IWT is calculated as the mean acceleration between slow and intermediate walking intensity during the last 30 seconds of these intervals in the fitness test. The fast walking cutoff intensity is calculated as the mean acceleration of the intermediate and fast walking intensity during the last 30 seconds of this interval in the fitness test. If the user exceeds these cutoffs during IWT, the audio guidance encourages the user to slow down (for slow walking) or speed up (for fast walking).

\section{IWT history and postsession feedback}

After terminating each IWT session or fitness test, the app displays data from the session performed (Figure 5D). Historical training data are also available (Figure 5E). These include information about walking distance in kilometers (calculated from GPS), number of steps, duration, and the quality of performance of the IWT sessions. The quality of an IWT session is determined as the fraction of training time (percentage), which the user has spent below (for slow walking) or above (for fast walking) the intensity cutoffs. This performance quality information is delivered to the user by four different smiley icons (Figure 6). 
Figure 6 Interpretation of the smiley feedback system. Abbreviation: IWT, interval walking training.

\section{Data logging and storage}

The epoch level accelerometer data and GPS coordinates during interval walking and the fitness test are immediately transferred to a central server using either WIFI or the mobile data network (100 bytes/s) after terminating each session. Data are also stored on the device for up to 8 days in case no network is available upon termination of a session. When the device connects to a network and the app is engaged, the recent locally stored data are then automatically transferred to the server. Accelerometer and GPS data are transferred along with the hashed CPR number and self-reported user data. The hash algorithm, called SHA-256, adds extra values to the CPR number and translates it into a string. The SHA256 is a one-way algorithm, that is, dehashing is only possible by comparing two encryption keys generated from identical CPR numbers. Other information in the InterWalk app is not hashed but cannot be connected to the CPR number. Stored data are described in Table 1.

Table I IWT and user data transferred to the server from the smartphone

\begin{tabular}{ll}
\hline Variables & Data source \\
\hline Acceleration $\left(\mathrm{m} / \mathrm{s}^{2}\right)($ vector magnitude) & Accelerometer \\
Geographical location & GPS \\
Intensity cutoffs $\left(\mathrm{m} / \mathrm{s}^{2}\right)$ & Accelerometer \\
Distance covered $(\mathrm{km})$ & GPS \\
Frequency of use & Upload \\
Training duration (min) & Accelerometer \\
Quality of IWT $(\%$ correct physical activity intensity) & Accelerometer \\
Steps & Accelerometer \\
Body height $(\mathrm{cm})$ & Self-report \\
Body weight $(\mathrm{kg})$ & Self-report \\
Sex & Self-report \\
CPR & Self-report \\
Name & Self-report \\
\hline
\end{tabular}

Abbreviations: IWT, interval walking training; GPS, Global Positioning System; CPR, Central Personal Registry.

\section{Dissemination of the InterWalk app - current status}

The InterWalk app was released into the general population via the iTunes App Store in November 2013 (updated March 2014) and on Google Play in October 2014. The app is free of charge. It is actively promoted by the Danish Centre for Strategic Research in Type 2 Diabetes (DD2, www.dd2.nu), the Danish Diabetes Association and partner municipalities through their websites, and at the InterWalk webpage www. interwalk.dk (in Danish).

Currently (92 weeks postrelease), 31,829 persons have downloaded the InterWalk app from the iTunes App Store $(\mathrm{N}=27,647)$ or Google Play $(\mathrm{N}=4,182)$. Of these, 9,466 persons $(30 \%)$ entered their CPR number and were included in the cohort. Table 2 describes the baseline characteristics upon cohort entry (first use of the app). Fewer men (29\%) downloaded and entered their CPR number than women (71\%). Generally, the male participants were older than the women (median age 60 years vs 54 years). Few participants were of normal weight, and men had a higher prevalence of overweight ( $41 \%$ vs $32 \%$ with body mass index [BMI] $25-29.9)$ and obesity (39\% vs $33 \%$ with BMI $\geq 30)$ than did women. Women more often downloaded the app on an iPhone mobile operating system compared to men, whereas men more often downloaded the app on an Android operating system (Table 2).

\section{Research plan}

The health impact of a nationwide implementation of health care mobile solutions such as the InterWalk app on the population with T2D is still uncertain. Researchers in Denmark will be able to retrieve a large number of outcome measures and confounding factors by taking advantage of linkage with the already existing health databases. This renders a unique opportunity to evaluate health impacts of releasing and implementing the InterWalk app into the Danish population with T2D. ${ }^{21}$

\section{Planned initial data analyses}

Data collected during InterWalk use will potentially enable researchers to answer important research questions on physical exercise promotion. Initially, nationwide data linkage and analysis may answer questions such as 1) What are the individual predictors, for example, age, sex, sociodemographic characteristics, comorbidities, of IWT uptake, that is, of using the InterWalk app and of its sustained usage? 2) Does the use of the InterWalk app affect the subsequent risk of incident hospitalizations with cardiovascular events, other 
Table 2 Sample characteristics at first registration

\begin{tabular}{|c|c|c|c|}
\hline & All & Men & Women \\
\hline $\mathrm{N}$ & $9,466(100 \%)$ & $2,784(29 \%)$ & $6,682(71 \%)$ \\
\hline Age (years) & $55.6(46.4-63.6)$ & $59.7(50.3-66.8)^{*}$ & $54.0(44.5-61.9)$ \\
\hline Body weight (kg) & $80.0(69.0-95.0)$ & $92.0(82.0-105.0)^{*}$ & $75.0(66.0-88.0)$ \\
\hline Body height $(\mathrm{cm})$ & $170.0(\mid 65.0-178.0)$ & $180.0(\mid 75.0-184.0)^{*}$ & $168.0(163.0-172.0)$ \\
\hline BMI & $27.4(24 .|-3| .6)$ & $28.4(25.6-32.2)^{*}$ & $26.7(23.6-31.2)$ \\
\hline \multicolumn{4}{|l|}{ Weight status (BMI $\left[\mathrm{kg} / \mathrm{m}^{2}\right]^{*}$ ) } \\
\hline Normal weight (BMI <25), N (\%) & $3,028(3 \mathrm{I} . \mathrm{I})$ & $568(20.4)$ & $2,475(37.1)$ \\
\hline Overweight (BMI $\geq 25$ and $<30$ ), $N(\%)$ & $3,310(34.9)$ & $\mathrm{I}, 144(4 \mathrm{I} . \mathrm{I})$ & $2,160(32.3)$ \\
\hline Obese (BMI $\geq 30$ ), N (\%) & $3,127(33.0)$ & $\mathrm{I}, 072(38.5)$ & $2,047(30.6)$ \\
\hline \multicolumn{4}{|l|}{ Mobile operating system* } \\
\hline iPhone operating system, N (\%) & $6,972(73.5)$ & $1,913(73.5)$ & $5,049(80.2)$ \\
\hline Android, N (\%) ${ }^{\mathrm{a}}$ & $\mathrm{I}, 799(19.0)$ & $692(26.5)$ & $\mathrm{I}, 10 \mathrm{I}(19.8)$ \\
\hline
\end{tabular}

Notes: Data are medians (interquartile range) or actual numbers (relative frequency). aSubsample of 8,77I users (only registered in Version I.I). $* P<0.00 \mathrm{I}$ for sex differences (Wilcoxon's signed rank test or Fisher's exact test).

Abbreviation: BMI, body mass index.

incident disease outcomes, and total mortality? 3) Does the use of the InterWalk app affect total health care expenditure and consumption?

\section{Use of external data sources}

The InterWalk data will be linked with data available in the DD2 project database. The DD2 project cohort (current $\mathrm{N}>6,900$ ) prospectively enrolls newly diagnosed patients with T2D from all Danish general practitioners and hospital clinics; it includes biobank data from blood and urine samples along with baseline examination data on, for example, self-reported physical activity, family history of T2D, body weight at age of 20 years, resting heart rate, and waist and hip circumference. Data on a range of other variables (eg, smoking, blood pressure, lipids, hemoglobin Alc, comorbidities, medications) are ascertained through data linkage with other databases. ${ }^{22}$ Furthermore, in a subgroup of the DD2 cohort, patients' quality of life and mental state are prospectively evaluated using the SF12 and WHO-5 questionnaires. ${ }^{23}$ The DD2 data set is linked with the National Danish Prescription Registry, the National Danish Patient Register, and the Civil Registration System providing continuous up-to-date information on all incident hospital contacts and mortality. ${ }^{24,25}$

All statistical analyses are performed at the DD2 data management center, Department of Clinical Epidemiology at Aarhus University Hospital, Denmark. The InterWalk project has been approved by the Danish Data Protection Agency (approval number 2008-58-0035). The Ethical Scientific Committee of Southern Denmark considers the InterWalk setup a health promoting initiative; thus, no written consent from the participants or further approval from the committee is needed for this study (S-20122000-77).

\section{Discussion}

The InterWalk app has now been freely available to the broad Danish population for $\sim 2$ years. This study provides a description of the InterWalk project and app and of the baseline characteristics of the persons who have entered the cohort with their CPR number during the first $\sim 2$ years.

The prevalence of InterWalk app users being either overweight (BMI $\geq 25$ ) or obese (BMI $\geq 30$ ) was higher compared to the Danish population of similar age (ages 45-74 years) with a prevalence odds ratio ( $95 \%$ confidence intervals) of 2.1 (1.9-2.3) for men and 1.8 (1.7-1.9) for women. ${ }^{26}$ For obesity (BMI $\geq 30$ ) only, the prevalence was also increased for both male and female app users, with a prevalence odds ratio (95\% confidence intervals) of 2.8 (2.6-3.0) for men and $2.2(2.1-2.3)$ for women as compared to representative Danish reference data (ages 45-74 years). ${ }^{26}$ The increased prevalence of overweight and obesity in the InterWalk cohort compared to the Danish reference population suggests that the InterWalk app is in fact of interest for the at-risk (overweight and obese) individuals in the population.

There is a societal interest in developing effective and sustainable (feasible and outcome-efficient) methods to promote physical activity for the general population, in particular for people with inactivity-associated diseases such as T2D. Using mobile technology in T2D self-management is of growing interest due to the feasibility of long-distance consultation and self-monitoring of the intervention. Besides being a T2D self-management tool for the individual patient, the InterWalk app offers municipalities and clinicians a readily available tool to promote individualized and unsupervised structured physical activity in a free-living setting. To succeed with implementation of mobile solutions such as the InterWalk app, knowledge is needed about factors motivating 
adherence. Knowledge is also needed on how additional transition into other settings of physical activity (eg, fitness centers or other mobile solutions) can be facilitated. Although primarily developed for patients with T2D, the InterWalk app could be used to promote physical activity in other patient groups, including "hard-to-reach" groups such as patients with cancer. However, more research on adaption to these groups is needed.

The development of the InterWalk app was done in collaboration between key stakeholders (patients, patient associations, and clinicians) in order to increase the acceptability, user-friendliness, and graphical design. Inclusion of these stakeholders is pivotal in increasing adherence to e-health solutions. ${ }^{27}$ We find it likely that the InterWalk app could be implemented to a degree that would impact overall health in populations with T2D; yet, this needs to be evaluated in future observational and randomized controlled trials.

As more knowledge is gained about the usage of the InterWalk app, additional information on motivational predictors of the app usage will also be collected, shedding light on the potential impact on quality of life in T2D. Furthermore, since almost $\sim 30,000$ Danes have already downloaded the InterWalk app, there is a large potential to reach even more people over time.

As we expect the InterWalk app to be used in the general population, not only in patients with $\mathrm{T} 2 \mathrm{D}$, analyses on the potential effectiveness on health outcomes in the nondiseased general population will also be possible in the future.

\section{Acknowledgments}

The authors wish to thank the foundations that support the work. The study is part of the DD2, which is the acronym for "The Danish Centre for Strategic Research in Type 2 Diabetes", that is supported by grants from The Danish Strategic Research Council (grant nos 09-067009 and 09-075724). All funding and partners are listed at www. dd2.nu. The Centre of Inflammation and Metabolism (CIM) is supported by a grant from the Danish National Research Foundation (DNRF55). The Centre for Physical Activity Research (CFAS) is supported by a grant from Trygfonden. The CopenRehab is supported by a grant from the Municipality of Copenhagen. Furthermore, this study is supported by a postdoc grant from The Danish Diabetes Academy (supported by the Novo Nordic Foundation) and a grant from the Augustinus Foundation. The authors would like to thank Dr Carsten Fallesen for technical support in the development of the InterWalk app.

\section{Author contributions}

Mathias Ried-Larsen, Reimar W Thomsen, and Jens S Nielsen drafted the manuscript. All authors contributed toward data analysis, drafting and critically revising the paper and agree to be accountable for all aspects of the work.

\section{Disclosure}

The authors report no conflicts of interest in this work.

\section{References}

1. Umpierre D, Ribeiro PA, Schaan BD, Ribeiro JP. Volume of supervised exercise training impacts glycaemic control in patients with type 2 diabetes: a systematic review with meta-regression analysis. Diabetologia. 2013;56(2):242-251.

2. Boule NG, Haddad E, Kenny GP, Wells GA, Sigal RJ. Effects of exercise on glycemic control and body mass in type 2 diabetes mellitus: a meta-analysis of controlled clinical trials. JAMA. 2001;286(10): $1218-1227$.

3. Colberg SR, Sigal RJ, Fernhall B, et al; American College of Sports Medicine; American Diabetes Association. Exercise and type 2 diabetes: the American College of Sports Medicine and the American Diabetes Association: joint position statement. Diabetes Care. 2010;33(12):e147-e167.

4. Umpierre D, Ribeiro PA, Kramer CK, et al. Physical activity advice only or structured exercise training and association with $\mathrm{HbAlc}$ levels in type 2 diabetes: a systematic review and meta-analysis. JAMA. 2011;305(17):1790-1799.

5. Shaw JE, Sicree RA, Zimmet PZ. Global estimates of the prevalence of diabetes for 2010 and 2030. Diabetes Res Clin Pract. 2010; 87(1):4-14.

6. Whiting DR, Guariguata L, Weil C, Shaw J. IDF diabetes atlas: global estimates of the prevalence of diabetes for 2011 and 2030. Diabetes Res Clin Pract. 2011;94(3):311-321.

7. Center PR. Smartphone Ownership - 2013 Update. Washington DC: Pew Research Center's Internet and American Life Project; 2013.

8. Denmark S. [Domestic electronics ownership 2013] Elektronik $i$ hjemmet 2013. Denmark; 2013. Available from: https://www.dst.dk/ $\mathrm{da} /$ Statistik/emner/forbrug/elektronik-i-hjemmet?tab=nog\#. Accessed Feb 22, 2016.

9. Boule NG, Kenny GP, Haddad E, Wells GA, Sigal RJ. Meta-analysis of the effect of structured exercise training on cardiorespiratory fitness in type 2 diabetes mellitus. Diabetologia. 2003;46(8):1071-1081.

10. Masuki S, Mori M, Tabara Y, et al; Shinshu University Genetic Research Consortium. Vasopressin V1a receptor polymorphism and interval walking training effects in middle-aged and older people. Hypertension. 2010;55(3):747-754.

11. Nemoto K, Gen-no H, Masuki S, Okazaki K, Nose H. Effects of highintensity interval walking training on physical fitness and blood pressure in middle-aged and older people. Mayo Clin Proc. 2007;82(7):803-811.

12. Nose H, Morikawa M, Yamazaki T, et al. Beyond epidemiology: field studies and the physiology laboratory as the whole world. J Physiol. 2009;587(pt 23):5569-5575.

13. Morikawa M, Okazaki K, Masuki S, et al. Physical fitness and indices of lifestyle-related diseases before and after interval walking training in middle-aged and older males and females. Br J Sports Med. 2011;45(3):216-224.

14. Yamazaki T, Gen-No H, Kamijo Y, Okazaki K, Masuki S, Nose H. A new device to estimate VO2 during incline walking by accelerometry and barometry. Med Sci Sports Exerc. 2009;41(12):2213-2219.

15. Karstoft K, Winding K, Knudsen SH, et al. The effects of free-living interval-walking training on glycemic control, body composition, and physical fitness in type 2 diabetic patients: a randomized, controlled trial. Diabetes Care. 2013;36(2):228-236. 
16. Pilemalm S, Timpka T. Third generation participatory design in health informatics - making user participation applicable to large-scale information system projects. J Biomed Inform. 2008;41(2):327-339.

17. Clemensen J, Larsen SB, Kyng M, Kirkevold M. Participatory design in health sciences: using cooperative experimental methods in developing health services and computer technology. Qual Health Res. 2007; 17(1):122-130.

18. Drivsholm TB, Snorgaard O. [Organization of treatment and control of type 2 diabetic patients]. Ugeskr Laeger. 2012;174(37):2159-2162. Danish.

19. Frank L. Epidemiology. When an entire country is a cohort. Science. 2000;287(5462):2398-2399.

20. Thygesen LC, Daasnes C, Thaulow I, Bronnum-Hansen H. Introduction to Danish (nationwide) registers on health and social issues: structure, access, legislation, and archiving. Scand J Public Health. 2011;39 (7 suppl):12-16.

21. Craig P, Cooper C, Gunnell D, et al. Using Natural Experiments to Evaluate Population Health Interventions: Guidance for Producers and Users of Evidence. London: Medical Research Council (MRC); 2011.
22. Nielsen JS, Thomsen RW, Steffensen C, Christiansen JS. The Danish centre for strategic research in type 2 diabetes (DD2) study: implementation of a nationwide patient enrollment system. Clin Epidemiol. 2012;4(suppl 1):27-36.

23. Pedersen KM, Andersen JS, Sondergaard J. General practice and primary health care in Denmark. J Am Board Fam Med. 2012;25(suppl 1):S34-S38.

24. Helweg-Larsen K. The Danish register of causes of death. Scand J Public Health. 2011;39(7 suppl):26-29.

25. Lynge E, Sandegaard JL, Rebolj M. The Danish national patient register. Scand J Public Health. 2011;39(7 suppl):30-33.

26. Christensen AI, Davidson M, Ekholm O, Pedersen PV, Juel K. Danskernes Sundhed. Den Nationale Sundhedsprofil 2013. København: [The National Health Profile 2013] Sundhedsstyrelsen; 2014. Danish.

27. van Gemert-Pijnen JE, Nijland N, van Limburg M, et al. A holistic framework to improve the uptake and impact of eHealth technologies. J Med Internet Res. 2011;13(4):e111.
Clinical Epidemiology

\section{Publish your work in this journal}

Clinical Epidemiology is an international, peer-reviewed, open access, online journal focusing on disease and drug epidemiology, identification of risk factors and screening procedures to develop optimal preventative initiatives and programs. Specific topics include: diagnosis, prognosis, treatment, screening, prevention, risk factor modification,

Submit your manuscript here: http://www.dovepress.com/clinical-epidemiology-journa

\section{Dovepress}

systematic reviews, risk \& safety of medical interventions, epidemiology \& biostatistical methods, and evaluation of guidelines, translational medicine, health policies \& economic evaluations. The manuscript management system is completely online and includes a very quick and fair peer-review system, which is all easy to use. 